\title{
Analysis of Optimal Operation Conditions for GaN-based Power Converters
}

\author{
Ander Avila ${ }^{a}$, Asier Garcia-Bediaga ${ }^{a}$, Alberto Rodriguez ${ }^{b}$, Luis $\operatorname{Mir}^{a}$, Alejandro Rujas ${ }^{a}$ \\ a) Power Electronics Area, IK4-IKERLAN Technological Research Centre, Arrasate-Mondragón, Spain, aavila@ikerlan.es \\ ${ }^{b)}$ Power Supply Systems Group (SEA), Universidad de Oviedo, Gijón, Spain, rodriguezalberto@uniovi.es
}

\begin{abstract}
The use of Gallium Nitride (GaN) devices allows breaking the performance barriers of conventional silicon-based (Si) low power converters by increasing the operating frequency while reducing system power losses. This paper evaluates the influence of the current ripple, switching frequency and number of GaN devices on the performance of a bi-directional buck converter. Furthermore, hard-switching and soft-switching operation modes are evaluated, identifying the optimal operation conditions for GaN-based power converters. Analytical and simulation models are employed and the obtained results are experimentally validated on a $3 \mathrm{~kW}$ GaN-based bi-directional buck converter.

Index Terms-Gallium Nitride, soft-switching, hard-switching
\end{abstract}

\section{INTRODUCTION}

The rapid evolution of power electronics results in higher efficiency and higher power density conversion systems. Until recent years, most of these power converters have been developed on silicon $(\mathrm{Si})$ power devices. Even if $\mathrm{Si}$ semiconductors present a good balance between performance and cost, the limits in terms of power density, operation temperature, and the switching frequency of Si-based switches are close to be reached [1]. Wide-bandgap (WBG) devices promise to break the performance barriers of conventional Si-based power converters, increasing operating frequency while reducing power losses [1]. In these terms, silicon carbide ( $\mathrm{SiC})$ and gallium nitride $(\mathrm{GaN})$ are the most promising materials because of their beneficial properties and current availability. $\mathrm{GaN}$ and $\mathrm{SiC}$ larger energy gap and better electric field characteristic than Si result in higher breakdown voltage capability for a thinner material. Consequently, the on-state resistance $\left(R_{d s, o n}\right)$ can be reduced, achieving lower conduction losses. Furthermore, although $\mathrm{Si}$ presents higher electron mobility than $\mathrm{SiC}$ and $\mathrm{GaN}$, the high saturation velocity of WBG devices results in an improvement high switching frequency operation [2].

While SiC devices have already been put into practice for high power applications [3], the trend of $\mathrm{GaN}$ devices has been move from microwave solutions [4] to power applications with higher voltage requirements $(>200 \mathrm{~V})$, in recent years. The interest of GaN-based transistors is mainly associated with the high electron mobility layer, also known as the twodimensional electron gas (2DEG). There are mainly three types

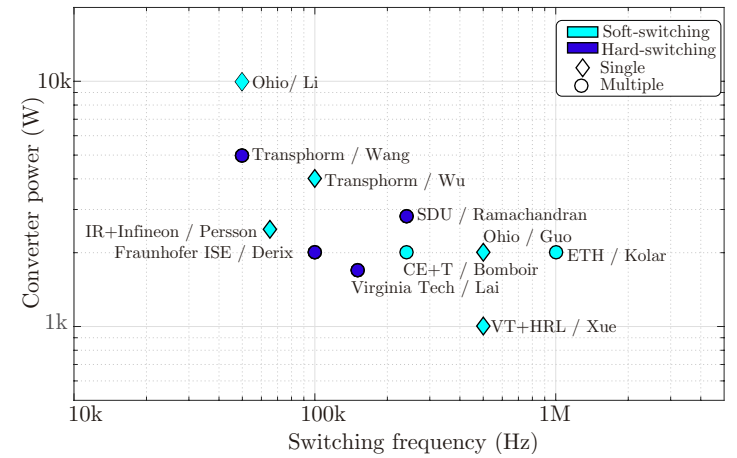

Fig. 1: Power vs. frequency map of GaN-based power converter prototypes proposed in literature.

of $\mathrm{GaN}$ switches available: GaN cascode, which is internally built with a low-voltage Si-MOSFET and normally on GaN; insulated gate high-electron-mobility transistor (HEMT); and non-insulated gate injection transistor (GIT).

Referring to GaN-based power converters, GaN devices have been implemented in topologies with soft-switching capability to operate at high switching frequencies $(200 \mathrm{kHz}$ $1 \mathrm{MHz}$ ) (see Fig. 1). Topologies such as dual active bridge [5], totem pole [6] or full-bridge [7] are proposed, achieving high power densities $(>8 \mathrm{~kW} / \mathrm{l})$ as it is presented in [8], [9]. Furthermore, in medium/high power converters $(>2 \mathrm{~kW})$, multiple GaN devices are paralleled [10], [11] or interleaved [8], [9], [12]-[14], usually with soft switching operation. However, there is not an established tendency for GaN devices for medium/high power solutions, as it can be observed from Fig. 1.

In this paper, the performance of a GaN-based bi-directional buck converter is evaluated in terms of the inductor current ripple, switching frequency and number of GaN devices connected in parallel. High inductor current ripples will be interesting for interleaved converters, reducing the output current ripple while achieving soft-switching transitions. The main contribution of the paper is the analysis of these design parameters and operation conditions to search the optimal implementation of $\mathrm{GaN}$ devices on medium/high power converters. The influence of this operation conditions is theoretically analyzed, and finally verified with experimental measurements. 
This work is organized as follows. Section II describes the static and dynamic characteristics of the selected GaN devices. Section III evaluates the design operation conditions influence on the performance of a GaN-based buck converter. Section IV presents the developed GaN-based buck converter prototype, which will be used to validate the results obtained in previous sections. Finally, Section V draws some conclusions about the influence of operation conditions on the performance of GaNbased power converters.

\section{GAN DEVICES FOR POWER APPLICATIONS}

The compact size of these devices makes difficult to operate in high current range ( $\geq 10 \mathrm{~A})$ without parallel-connected devices, in spite of $60 \mathrm{~A}$ announced $\mathrm{GaN}$ devices $\left(47 \mathrm{~A} @ 100^{\circ} \mathrm{C}\right.$ ). Thus, it is essential to identify the impact of parallelization and operation conditions on the characteristics of $\mathrm{GaN}$ devices. For that purpose, a general theoretical analysis is presented for a bi-directional buck converter (see Fig. 2), along with a performance analysis of $\mathrm{GaN}$ devices.

\section{A. Thermal management}

$\mathrm{GaN}$ devices require complex thermal management to extract the heat from such small thermal pad [15]. The device is assumed to be mounted on a heatsink (see Fig. 2), which is at $T_{h s}$ temperature, via thermal interface material (TIM). Then, the losses limit $P_{d, l i m}$ of semiconductors is obtained evaluating junction-to-case $\left(R_{t h_{j-c}}\right)$ and case-to-heatsink $\left(R_{t h_{c-h s}}\right)$ thermal resistances.

$$
P_{d, l i m}=\frac{T_{j, \max }-T_{h s}}{R_{t h_{j-c}}\left(A_{e}\right)+R_{t h_{c-h s}}\left(A_{e}\right)}
$$

being $T_{j, \max }$ the semiconductor junction temperature limit.

Moreover, $R_{t h_{j-h s}}$ and $R_{t h_{c-h s}}$ are inversely proportional to the effective area $\left(A_{e}\right)$ of devices, which increases with the number of parallel devices $\left(N_{p}\right)(2)$

$$
\begin{array}{r}
R_{t h_{j-c}}\left(A_{e}\right)=r_{t h_{j-c}} /\left(A_{e} \cdot N_{p}\right) \\
R_{t h_{c-h s}}\left(A_{e}\right)=r_{t h_{c-h s}} /\left(A_{e} \cdot N_{p}\right)
\end{array}
$$

where $r_{t h_{j-c}}$ and $r_{t h_{c-h s}}$ are junction-to-case and case-toheatsink specific thermal resistance $\left(\mathrm{mm}^{2} \mathrm{~K} / \mathrm{W}\right)$, respectively.

Considering $\mathrm{GaN}$ devices with low stray inductance package, i.e. small size packages, the impact of thermal resistances is too high, reducing the thermal cooling capability. Nevertheless, the use of $N_{p}$ number of devices increases the power dissipation capability for a junction-to-heatsink specific resistance $\left(r_{t h_{j}-h s}\right)$

$$
P_{d, l i m}=\frac{T_{j, \max }-T_{h s}}{r_{t h_{j-h s}}} \cdot\left(A_{e} \cdot N_{p}\right)
$$

Hence, optimum number of devices has to be considered to satisfy the maximum allowed semiconductor losses for a $T_{j, \max }$ design constraint and the minimum power losses.

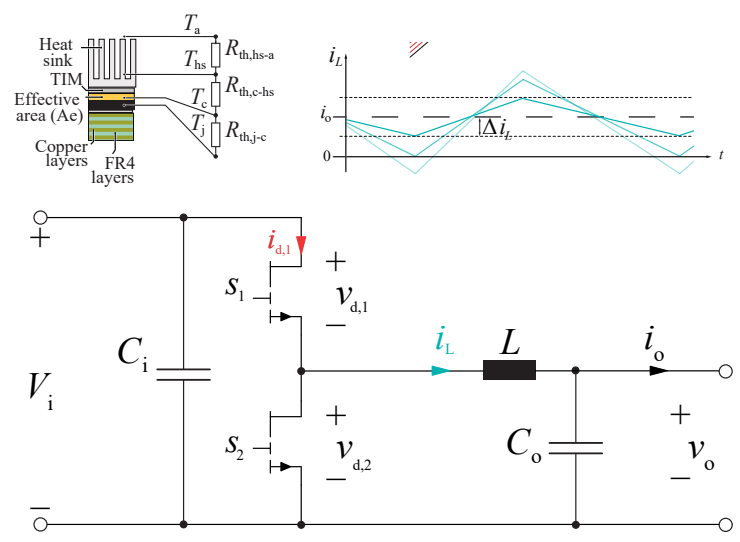

Fig. 2: GaN-based bi-directional buck converter thermal and electrical circuit.

\section{B. Power losses distribution}

Regarding power losses of switches, it is a well-known fact that conduction losses are scaled down with $N_{p}$ number of switches, resulting in lower conduction losses according to (4).

$$
P_{c}=I_{d, r m s}^{2} \cdot R_{d s, o n}\left(T_{j}\right) / N_{p}
$$

where $I_{d, r m s}$ is the device RMS current, which is dependent on the average current $I_{o}$ and current ripple $\Delta i_{L}$ (5).

$$
I_{d, r m s}=\sqrt{I_{o}^{2}+\Delta i_{L}^{2} / 3}
$$

Moreover, during dead-time $t_{d t}$ power switches are reverse conducting, incurring in losses, which can be a limiting factor if the ratio between dead-time and switching period is relevant.

$$
P_{d t}=\left(v_{s d, o n} I_{o n}+v_{s d, o f f} I_{o f f}\right) t_{d t} f_{s}
$$

where $v_{s d}$ is the reverse drain-to-source voltage drop for the switching currents $\left(I_{o n}\right.$ and $\left.I_{o f f}\right)$. These losses will be also relevant for devices with high $v_{s d}$.

Furthermore, considering a triangular current with an average current $I_{o}$ (see Fig. 2), $I_{o n}$ decreases with the current ripple, while $I_{o f f}$ switching current increases (7). Thus, the current ripple has not a great influence on total power losses, considering that the reverse voltage variation is not important.

$$
\begin{gathered}
I_{o n}=I_{o}-\Delta i_{L} \\
I_{o f f}=I_{o}+\Delta i_{L}
\end{gathered}
$$

Switching losses for a hard-switching transition consist of turn-on and turn-off losses (8), which are proportional to switching frequency $f_{s}$ and can be scaled by the switched voltage $V_{d}$ for a reference switching voltage $V_{d, \text { ref }}$ [16].

$$
P_{s}\left(V_{d}\right)=\left[E_{o n}\left(I_{o n}, V_{\text {ref }}\right)+E_{\text {off }}\left(I_{o f f}, V_{\text {ref }}\right)\right] \cdot f_{s} \cdot \frac{V_{d}}{V_{\text {ref }}}
$$

where turn-on $\left(E_{o n}\right)$ and turn-off $\left(E_{o f f}\right)$ energies are dependent on switching currents $I_{o n}$ and $I_{o f f}$, respectively. 
An approximation of $E_{o n}$ and $E_{\text {off }}$ for a given $V_{d}$ voltage can be made using a second order polynomial, considering $N_{p}$ number of devices.

$$
\begin{array}{r}
E_{s}\left(V_{d}\right)=\left(c\left(V_{d}\right)\right. \\
\left.\cdot\left(\frac{I_{s}}{N_{p}}\right)^{2}+b\left(V_{d}\right) \cdot \frac{I_{s}}{N_{p}}+a\left(V_{d}\right)\right) \cdot N_{p} \\
=\frac{c\left(V_{d}\right)}{N_{p}} \cdot I_{s}{ }^{2}+b\left(V_{d}\right) \cdot I_{s}+a\left(V_{d}\right) \cdot N_{p}
\end{array}
$$

being $I_{s}$ the switching current, $I_{o n}$ for turn-on and $I_{o f f}$ for turn-off transient.

Turn-on main losses of $\mathrm{GaN}$ devices are related to output capacitance self charging/discharging $\left(E_{o s s}\left(V_{d}\right)\right)$, which are only dependent on $V_{d}$, and the overlapping of switching voltage $v_{d}$ and switching current $i_{d}\left(E_{v i}\left(V_{d}, I_{o n}\right)\right)$ [16]. Reverse recovery energy is negligible for $\mathrm{GaN}$ devices, being one of the most relevant characteristic to reduce the impact of turnon losses on hard-switching operation. Hence, considering this turn-on loss distribution and the second order approximation (9), the term which is independent of the current $\left(a_{o n}\left(V_{d}\right)\right)$ is obtained (10)

$$
\begin{gathered}
a_{o n}\left(V_{d}\right) \approx Q_{o s s}\left(V_{d}\right) V_{d} \\
\text { where } Q_{o s s}\left(V_{d}\right)=\int_{0}^{V_{d}} C_{o s s}\left(v_{d}\right) d v_{d}
\end{gathered}
$$

being $C_{o s s}\left(v_{d}\right)$ the voltage-dependent parasitic output capacitance, which is the same for high-side and low-side devices. On the contrary, as turn-off transient does not present zerocurrent losses $\left(a_{o f f}\left(V_{d}\right) \approx 0 \mu J\right)$, all turn-off losses are related to the $v_{d}$ and $i_{d}$ overlapping.

Moreover, an increase of the current ripple can result on soft-switching operation. If the current ripple is $100 \%$ of $I_{o}$, zero-current-switching (ZCS) is achieved, being switching losses only related to the output capacitance $\left(E_{o s s}\right)$. Besides, if the current ripple is increased until $I_{o n}$ is negative zerovoltage-switching (ZVS) transition can be accomplished, as long as ZVS operation conditions are fulfilled.

\section{Conditions for soft-switching}

For ZVS operation it is crucial to specify the conditions under which the ZVS can be achieved. This requires a minimum current $\left(I_{o n, \min }\right)$ of the inductive component $(L)$, that charges/discharges output capacitances within the dead-time. The required energy of the inductance for ZVS can be derived for the energy balance (11).

$$
\frac{1}{2} L I_{o n, \min }^{2}>\frac{1}{2} N_{p}\left(C_{o s s_{1}}+C_{o s s_{2}}\right) V_{d}^{2}
$$

where $C_{o s s_{1}}$ is the high-side switch capacitance and $C_{o s s_{2}}$ the low-side switch capacitance.

Moreover, a resonant analysis of the circuit is performed, in order to define ZVS conditions more precisely during the dead-time. In a buck converter, ZVS is achieved if the switch node voltage of the top device $\left(v_{d 1}\right)$ excess input voltage $V_{i}$, turning-on the body diode of $s_{1}$ (see Fig. 2). Then, the minimum current for a ZVS condition is obtained by (12) within a minimum dead-time [17]

$$
\begin{gathered}
I_{o n, \min }>\sqrt{\frac{\left(C_{o s s_{1}}+C_{o s s_{2}}\right) V_{i}\left(V_{i}-2 V_{o}\right) N_{p}}{L},} \\
t_{d t, \text { min }}=\frac{1}{\omega_{o}}\left(\tan ^{-1}\left(\frac{V_{o}}{I_{o n, m i n}}\right)+\frac{\pi}{2}\right) .
\end{gathered}
$$

However, if conditions presented before are not fulfilled, incomplete ZVS occurs. This means that the top side switch turns-on before the resonant transition with a voltage $\Delta V_{d}$. As it is analyzed in [18] $\Delta V_{d}$ can be calculated solving the energy balance (11) and then obtaining the delivered energy for the incomplete ZVS

$$
E_{\Delta V}=N_{p} \cdot V_{d} \cdot\left(Q_{o s s}\left(V_{d}\right)-Q_{o s s}\left(V_{d}-\Delta V_{d}\right)\right)
$$

\section{Performance analysis}

In this paper the performance of $600 \mathrm{~V} 30 \mathrm{~A} \mathrm{GaN} \mathrm{GIT} \mathrm{de-}$ vices are analyzed using SPICE models. Forward and reverse conduction characteristics present low conduction resistance for forward conduction but not for open state reverse conduction [see Fig. 3(a)]. Free-wheeling reverse conduction of GaN devices have a higher reverse voltage drop, leading to specially consider an optimal dead-time which minimizes this effect for high-switching frequency operation. Regarding the conduction resistance of the analyzed devices, the analyzed GaN device shows up a low conduction resistance with a positive thermal coefficient, as it is depicted in Fig. 3(a). Thus, in case of paralleling devices, the positive thermal coefficient enables a natural balancing of current sharing through devices.

Switching losses are obtained integrating the $\left(v_{d}(t) \cdot i_{d}(t)\right)$ over turn-on and turn-off switching times. Figure 3(b) presents energy curves for different switching voltages. Considering turn-on and turn-off approximation terms (9), switching energy $E_{s}$ per device is obtained as a function of $\Delta i_{L}$ and $N_{p}$.

$$
\begin{gathered}
E_{s}=\Delta i_{L}^{2} \frac{c_{o f f}+c_{o n}}{N p}+ \\
\Delta i_{L}\left(2 I_{o} \frac{c_{o f f}-c_{o n}}{N_{p}}+b_{o f f}-b_{o n}\right)+\gamma
\end{gathered}
$$

being $\gamma$ the factor which is independent of the current ripple.

$$
\gamma=N_{p} Q_{o s s} V_{d}+\frac{I_{o}^{2}\left(c_{o f f}+c_{o n}\right)}{N_{p}}+I_{o}\left(b_{o f f}+b_{o n}\right)
$$

As it can be deduced from Fig. 3(b), turn-on and turn-off energy curves present a linear tendency being $b_{o n}>>c_{\text {on }}$ and $b_{o f f}>c_{o f f}$. Then, the total switching losses can be approximated to

$$
E_{s} \approx \underbrace{\left(I_{o}-\Delta i_{L}\right) b_{o n}+N_{p} Q_{o s s} V_{d}}_{E_{o n}}+\underbrace{\left(I_{o}+\Delta i_{L}\right) b_{o f f}}_{E_{o f f}}
$$



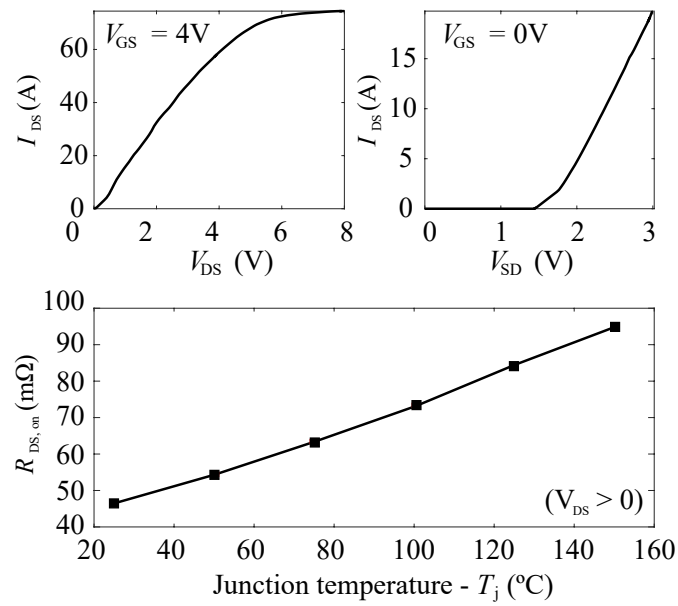

(a)
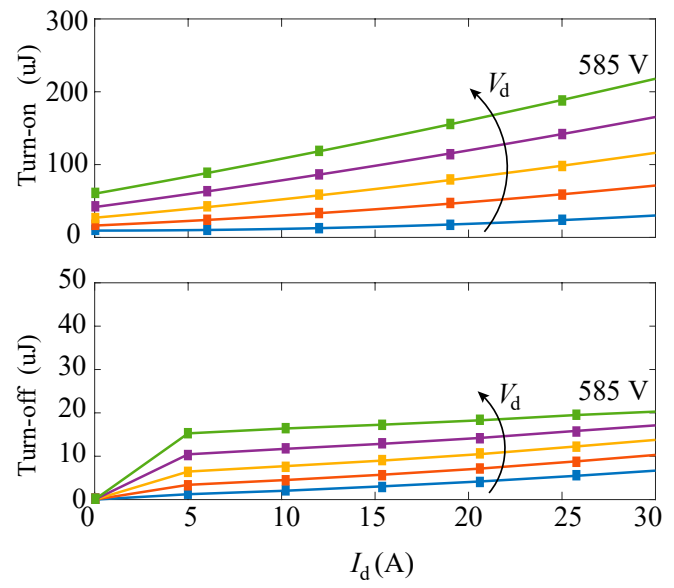

(b)

Fig. 3: GaN GIT device characteristics analysis, based on SPICE models. (a) Forward conduction and reverse open state $\left(V_{G S}=0 \mathrm{~V}\right)$ conduction characteristic, along with on-state resistance at different junction temperatures. (b) Switching energies at different switching voltages $\left(V_{d}\right)$.

Besides, the total switching losses increment $\Delta E_{s}$ as a function of $\Delta i_{L}$ is derived from (17).

$$
\Delta E_{s} \approx \Delta i_{L}\left(b_{o f f}-b_{o n}\right)
$$

Thus, if $b_{o n}$ is bigger than $b_{o f f}$, total switching energies are reduced when $\Delta i_{L}$ increases (18). This assumption is valid for $\mathrm{GaN}$ switches, which presents higher turn-on than turn-off losses, as it is compared in Fig. 3(b). Furthermore, the switching losses also increase with the output capacitance losses for $N_{p}$ number of devices (17).

In addition, total power losses difference is obtained as a function of $\Delta i_{L}$ and $N_{p}$ for the same $I_{o}(19)$.

$$
\begin{array}{r}
P_{d}=\frac{R_{d s, o n}\left(T_{j}\right)}{N_{p}} \frac{\Delta i_{L}^{2}}{3} \\
+f_{s}\left[\Delta i_{L}\left(b_{o f f}-b_{o n}\right)\right]+N_{p} Q_{o s s} V_{d}
\end{array}
$$

An increase of the current ripple results on a reduction of turn-on losses, being possible to achieve ZVS operation. This feature will be interesting for high-switching operation. Nevertheless, with higher current ripple turn-off losses increase, this will be a limiting factor for power devices with high $b_{\text {of } f}$ (??). In addition, the RMS current, and thus conduction losses, also increase with the current ripple, being relevant especially when working at low switching frequencies (19).

Regarding parallelization, the use of parallel devices reduces conduction losses while increasing switching losses. Then, when conduction losses are the most relevant factor for power losses, paralleling devices is presented as the most suitable solution. Besides, the use of parallel-connected devices is also essential for high-switching frequencies, due to the limited thermal cooling capability of such small devices. Thus, in order to increase the cooling capability paralleled devices are used to increase the power losses limit (??).

Thus, a trade-off between the power dissipation capability (3) and power losses (19) has to be considered when choosing $N_{p}$ number of devices. A more detailed evaluation is presented considering the influence of these parameters $\left(\Delta i_{d}, f_{s}, N_{p}\right.$, $t_{d t}$ ) on converter level performance.

\section{GAN-BASED POWER CONVERTER DESIGN EVALUATION}

The performance of a GaN-based bi-directional buck converter is evaluated for the specifications shown in Table I. The influence of parallelization, current ripple, switching frequency and dead-time of GaN devices is analyzed in this section. Namely, semiconductors devices and inductive components have to be considered for the estimation of power losses, assuming ceramic capacitors with negligible power losses as input and output capacitors $\left(C_{i}, C_{o}\right)$.

Semiconductors losses are evaluated for an evenly distributed power losses with a conduction resistance at a junction temperature of $120^{\circ} \mathrm{C}$. Regarding switching losses, characteristics obtained from the SPICE model [see Fig. 3(b)] are considered, along with the analytical expressions for softswitching operation (14).

The current ripple of a buck converter is related to the output inductance $L$, which is defined by (20):

$$
\Delta i_{L}=\frac{V_{i}(1-\delta) \delta}{2 L f_{s}}
$$

where $V_{i}$ is the input voltage and $\delta$ the duty cycle, providing $\delta=0.5$ the maximum current ripple.

\section{A. Analysis conditions}

An analysis of the current ripple and switching frequency influence has been performed comparing single device with two parallel-connected devices.

For the current ripple analysis, a switching frequency of $100 \mathrm{kHz}$ and an output current of $10 \mathrm{~A}$ with a duty cycle of 0.7 


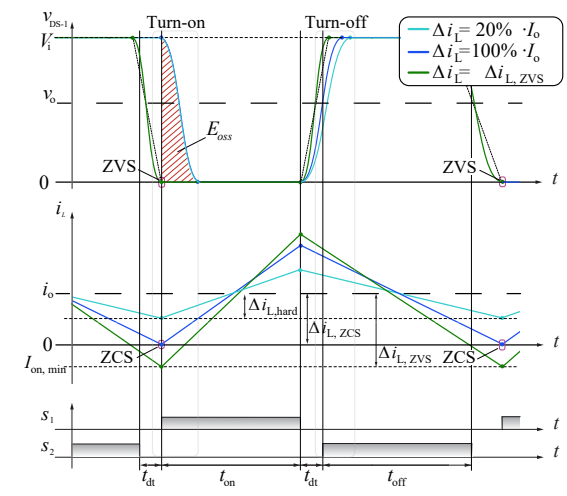

(a)

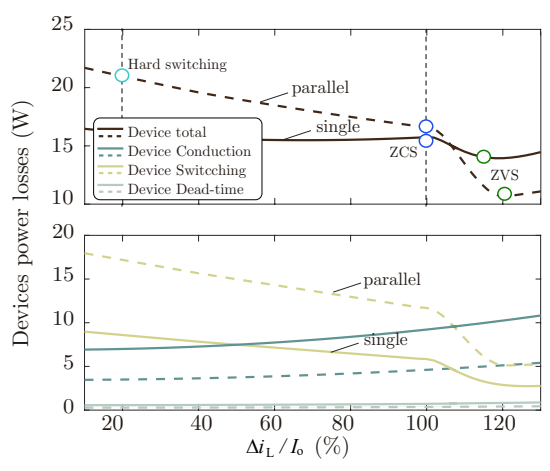

(b)

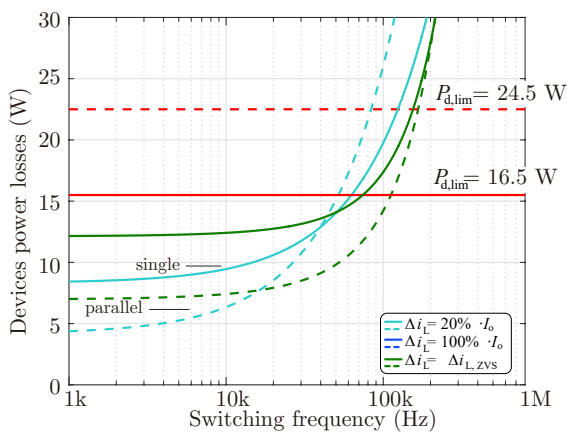

(c)

Fig. 4: Current ripple and switching frequency influence on a GaN-based bi-directional buck converter: (a) $20 \%, 100 \%$ and $\frac{\Delta i_{L}, Z V S}{I_{O}}$ comparison, achieving hard switching, ZCS and ZVS, respectively $\left(I_{o}=10 \mathrm{~A}\right)$. Analysis of devices power losses, comparing single with parallel-connected (dashed lines) GaN devices: (b) varying the current ripple and (c) for different switching frequencies, evaluating the thermal dissipation limit $\left(P_{d, l i m}\right)$.

TABLE I: Bi-directional buck electrothermal specifications.

\begin{tabular}{lccc}
\hline Description & Symbol & Value & Unit \\
\hline \hline Input voltage & $V_{i}$ & 350 & $\mathrm{~V}$ \\
Output voltage & $V_{o}$ & 245 & $\mathrm{~V}$ \\
Output current & $I_{o}$ & 10 & $\mathrm{~A}$ \\
Ambient, junction temperature & $T_{a}, T_{j}$ & 25,120 & ${ }^{\circ} \mathrm{C}$ \\
Heat-sink-to-ambient resistance & $R_{t h, h-a}$ & 2.25 & ${ }^{\circ} \mathrm{C} / \mathrm{W}$ \\
Case-to-heat-sink resistance & $R_{t h, c-h}$ & 2.9 & ${ }^{\circ} \mathrm{C} / \mathrm{W}$ \\
Junction-to-case resistance & $R_{t h, j-c}$ & 1 & ${ }^{\circ} \mathrm{C} / \mathrm{W}$ \\
\hline
\end{tabular}

are considered. Then, varying the output inductance, different current ripples are achieved (20). Low current ripple (20\%) presents hard switching for all the commutations (turn-on and turn-off) whereas the current ripple of $100 \%$ reduces turn-on switching losses, due to ZCS condition, as it is depicted in Fig. 4(a). A current ripple higher than $100 \%$ reduces even more these losses for specific conditions. A dead-time which ensures ZVS operation for the turn-on current (12) is selected.

Hence, the increase of the current ripple results in different operation modes defining three operation areas: hard switching $\left(\frac{\Delta i_{L}}{I_{o}}<100 \%\right), \operatorname{ZCS}\left(\frac{\Delta i_{L}}{I_{o}}=100 \%\right)$, and $\operatorname{ZVS}\left(\frac{\Delta i_{L, Z V S}}{I_{o}}\right)$ [see Fig. 4(a)].

Switching frequency influence is also evaluated under the same operation conditions for the three current ripples which provide three different operation modes: 20\%, 100\% and $>100 \%$.

\section{B. GaN devices performance}

Analyzing the power losses of $\mathrm{GaN}$ devices, the use of parallel devices for low current ripple results in higher power losses, due to the high relevance of switching losses, as it is depicted in Fig. 4(b). Nevertheless, as current ripple increases, switching losses are reduced and then the use of parallel devices reduces total power losses. For the frequency analysis, as $100 \%$ current ripple presents similar performance than low current ripple, only two current ripples are depicted in Fig. 4(c). The increase of the current ripple presents better performance than low current ripple approach, when paralleling devices or/and at high-switching frequencies.

In addition, the power losses limit of single device and paralleled devices is obtained, solving (3) for the specifications presented in Table I. Paralleling GaN switches reduce power losses for low switching frequencies, due to conduction losses reduction(4). However, for higher switching frequencies, although paralleling devices increase power losses, paralleling could be also interesting in high switching frequency due to higher power dissipation capability [see Fig. 4(c)].

\section{Power converter performance}

Power devices conduction losses and inductance losses increase with the current ripple, as it is presented in Fig. 4(b). However, switching losses are reduced mainly due to lower turn-on switching currents and the great difference between turn-on and turn-off energy of GaN devices (18). Results show that, for these specifications, the lowest power losses are achieved for $\Delta i_{L}=\Delta i_{L, Z V S}$. Figure 4(c) shows that for low switching frequencies $(<30 \mathrm{kHz})$, hard-switching operation presents the lowest power losses due to low current ripple, reducing the high impact of RMS current. However, as the switching frequency increases, switching losses become relevant, being $\Delta i_{L, Z V S}$ the most interesting operation point due to the impact of the output capacitance losses on $\mathrm{GaN}$ devices.

\section{EXPERIMENTAL RESULTS}

The GaN-based bi-directional buck converter presented in [15] is used to validate the results obtained in previous sections. This converter is composed by a power board based 


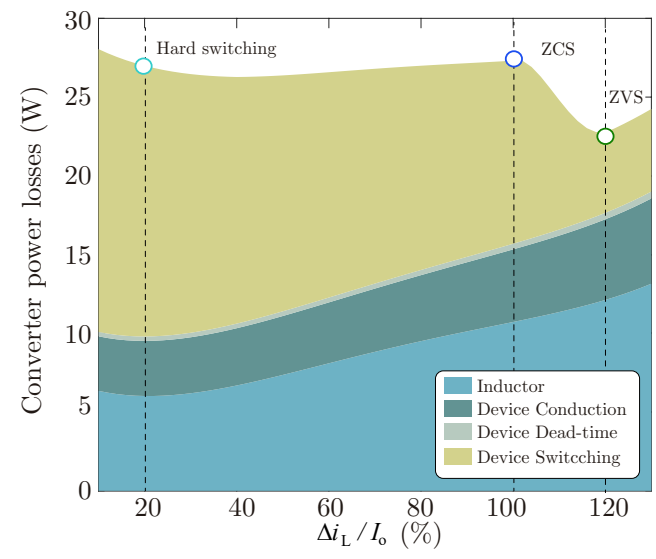

(a)

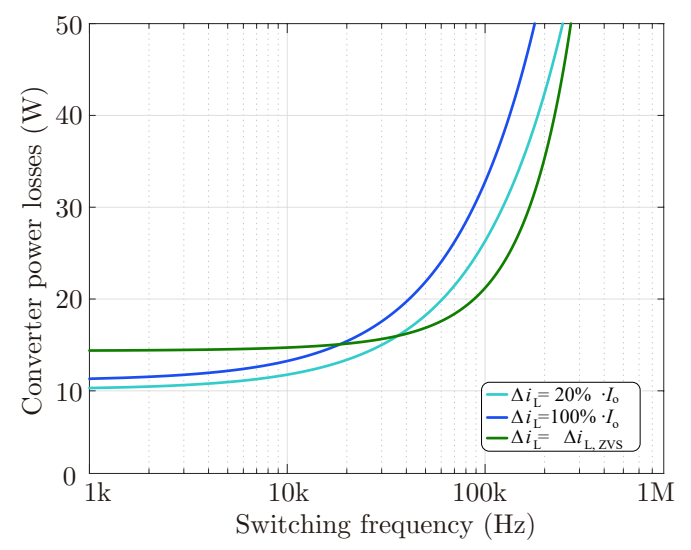

(b)

Fig. 5: Power converter performance analysis: (a) distribution of power losses for different current ripples $\left(\Delta i_{L} / I_{o}\right)$ and (b) comparison of buck converter power losses, varying the switching frequency for the three current ripples.

on two paralleled GaN GIT devices. The gate-loop length is equal for paralleled $\mathrm{GaN}$ devices, providing identical turn-on and turn-off times for the two parallel devices. Identical gate resistances circuits are employed in order to drive parallelconnected devices from the same driver and control, keeping a good thermal balancing. Thermography of the power converter under operation shows that there is a minor temperature difference between paralleled devices [see Fig. 6(b)]. Regarding the thermal management, the power board is cooled using PCB capabilities, $\mathrm{Cu}$ inlay and heat spreading, achieving a total power dissipation limit of $25.6 \mathrm{~W}$ [15].

\section{A. Test setup}

In order to validate the theoretical analysis, the influence of different current ripples on power devices is experimentally measured. For a switching frequency of $20 \mathrm{kHz}$ three output inductors are designed to compare the performance of three current ripples. In Fig. 7 the voltage of top device is depicted for three current ripples: $20 \%, 100 \%$ and $>100 \%$. Low current

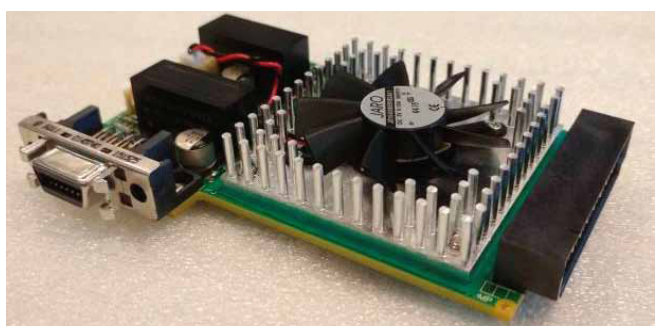

(a)

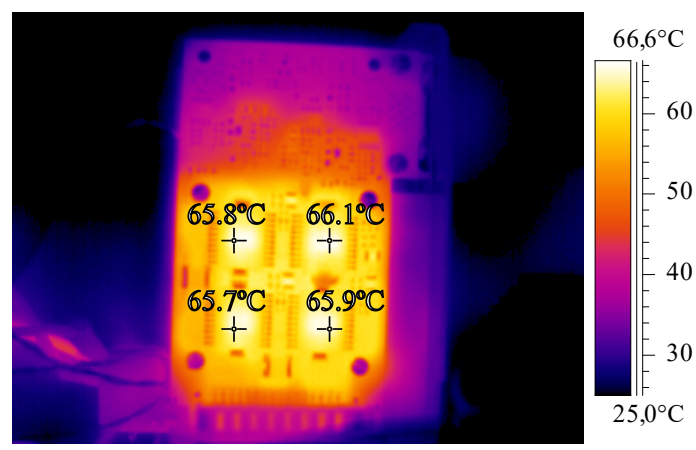

(b)

Fig. 6: GaN-based power converter prototype presented in [15]: (a) modular GaN-based bi-directional buck converter and (b) thermal distribution of $\mathrm{GaN}$ switches.

ripple presents a hard-switching transition, as it is depicted in Fig. 7(a). For the $100 \%$ current ripple the switch-node voltage is not discharged and thus, a hard-switching transition with ZCS is shown in Fig. 7(b). On the contrary, for the ZVS current ripple, the depicted $v_{d 1}$ shows up a complete soft-switching when turning-on the power switch [see Fig. 7(c)].

For the estimation of experimental power losses, heatsink and ambient temperature are measured together with input and output power values. Devices power losses $\left(P_{d, l o s s}\right)$ are obtained from the thermal equivalent heat-sink resistance $\left(R_{t h_{h s-a}}\right)$ and the measured heatsink-to-ambient temperature difference $\left(\triangle T_{h s}=T_{h s}-T_{a}\right)$ (21), when the thermal equilibrium is achieved

$$
P_{d, l o s s}=\frac{\triangle T_{h s}}{R_{t h_{h s-a}}} .
$$

In order to obtain the precise thermal resistance, the measured point has to be calibrated by measurements. Hence, a constant controlled current $\left(I_{d}\right)$ is fed into the power board, measuring $\triangle T_{h}$ for different controlled power levels. Then, power losses for a measured $\triangle T_{h s}$ are obtained [see Fig. 8(a)]. In addition, the voltage drop of devices has also been measured, in order to obtain $R_{d s_{o n}}\left(T_{j}\right)$ and thus, the junction temperature $T_{j}$ is estimated from the Fig. 3(a). The measured $P_{d, l o s s}$ consist of conduction $P_{c}$, switching $P_{s}$ and $P_{d t}$ dead-time power losses of devices. Measuring the RMS current of those switches $\left(I_{d, R M S}\right)$ and with the $R_{d s_{o n}}\left(T_{j}\right)$, 


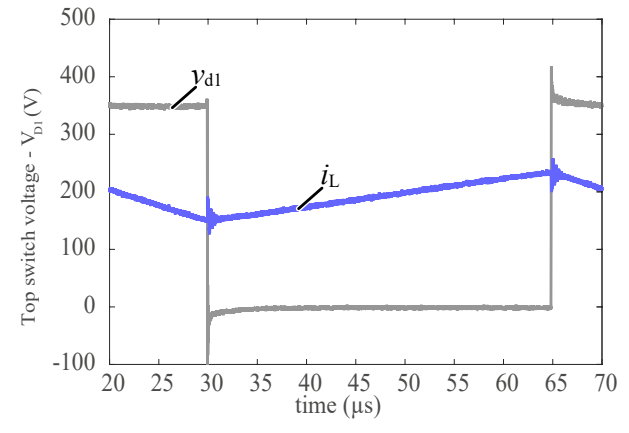

(a)

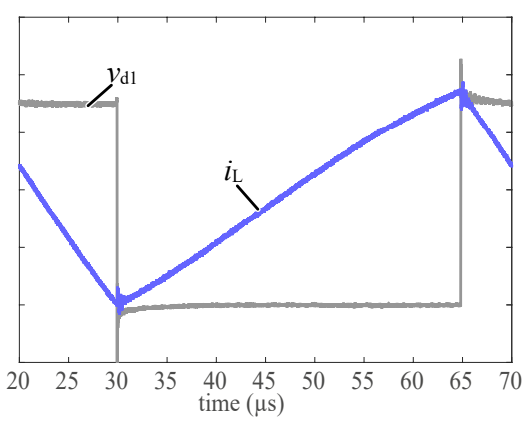

(b)

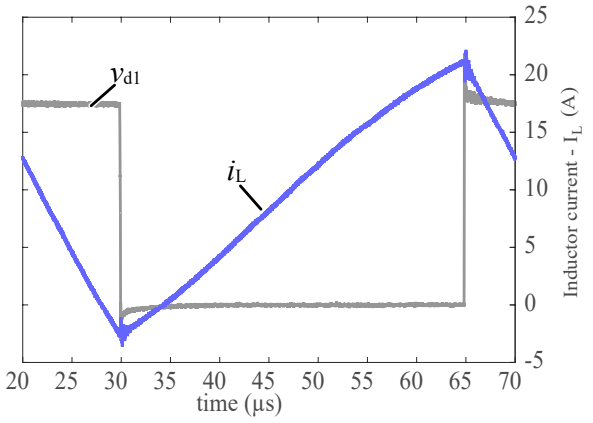

(c)

Fig. 7: Experimental measurements with different current ripples: (a) $20 \%$, (b) $100 \% \mathrm{z}$ and (c) $\frac{\Delta i_{Z V S}}{I_{o}}$.

conduction losses are known and hence the switching losses plus dead-time losses are calculated

$$
P_{s}+P_{d t}=P_{d, l o s s}-R_{D S_{o n}}\left(T_{j}\right) \cdot I_{d, R M S}^{2}
$$

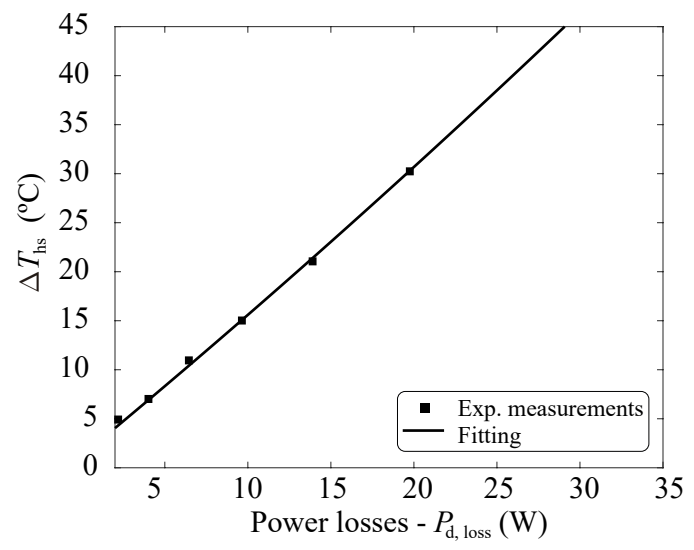

(a)

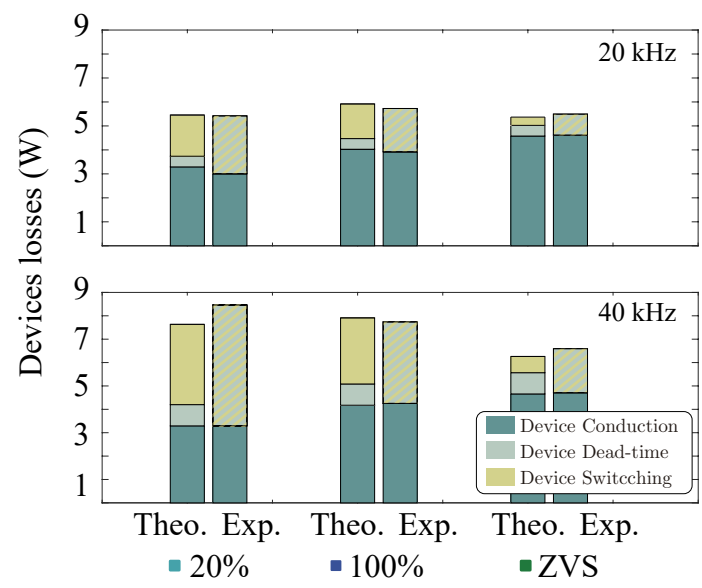

(b)

Fig. 8: Analysis of devices power losses, comparing different current ripples: (a) relation between heatsink temperature increment and power losses and (b) distribution of devices power losses $\left(I_{o}=10 \mathrm{~A} / V_{i}=350 \mathrm{~V}\right)$.

\section{B. Measurement results}

In Fig. 8(b) a good match between theoretical and experimental measurements is observed with a difference mainly related to the conduction losses. Hence, it is demonstrated how even for such low switching frequency (see Fig. 8(b)$20 \mathrm{kHz}$ ) the influence of the switching losses is reduced as the current ripple increases. However, the increase of the conduction losses leads to achieve similar power losses. Furthermore, when switching losses are more relevant (see Fig. 8(b)- $40 \mathrm{kHz}$ ) the increase of the current ripple results on even lower power losses [see Fig. 8(b)].

\section{Conclusions}

Although GaN switches present low power losses the complexity to extract the heat from such small devices limits the use of these devices in power applications. This paper analyzes the influence of different operation conditions in order to reduce $\mathrm{GaN}$ devices power losses and increase thermal cooling capability. On the one hand, the increase of the current ripple reduces power losses for high switching frequencies $(>40 \mathrm{kHz})$. The great difference between turn-on and turnoff energies, along with low conduction resistance of $\mathrm{GaN}$ devices leads to a reduction of devices losses. Moreover, the increase of the current ripple, ensuring ZVS avoids turn-on losses and allows high switching frequency operation. On the other hand, the use of parallel connected devices results in higher thermal limit, being possible to achieve higher output currents. Then, parallel-connected devices are not only used to reduce the power losses at low switching frequencies but also to increase the thermal cooling capability. Hence, the use of parallel-connected devices will be essential for medium/high power converters.

\section{REFERENCES}

[1] J. Millan, P. Godignon, X. Perpina, A. Perez-Tomas, and J. Rebollo, "A Survey of Wide Bandgap Power Semiconductor Devices," IEEE Transactions on Power Electronics, vol. 29, no. 5, pp. 2155-2163, May 2014. 
[2] E. A. Jones, F. F. Wang, and D. Costinett, "Review of Commercial GaN Power Devices and GaN-Based Converter Design Challenges," IEEE Journal of Emerging and Selected Topics in Power Electronics, vol. 4, no. 3, pp. 707-719, Mar. 2016.

[3] A. Rujas, V. M. Lpez, A. Garca-Bediaga, A. Berasategi, and T. Nieva, "Influence of $\mathrm{SiC}$ technology in a railway traction DC-DC converter design evolution," in IEEE Energy Conversion Congress and Exposition (ECCE), 2017.

[4] M. W. Lee, Y. S. Lee, and Y. H. Jeong, "A high-efficiency GaN HEMT hybrid class-E power amplifier for $3.5 \mathrm{GHz}$ WiMAX applications," in European Microwave Conference (EuMC), 2008.

[5] L. Xue, Z. Shen, M. Mu, D. Boroyevich, R. Burgos, B. Hughes, and P. Mattavelli, "Bi-directional PHEV battery charger based on normallyoff GaN-on-Si multi-chip module," in Applied Power Electronics Conference and Exposition (APEC), 2014.

[6] Eric Persson, "Professional Education Seminar S17 GaN HEMTs in Power Electronics," in Applied Power Electronics Conference and Exposition (APEC), 2015.

[7] F. Guo, L. Fu, H. Li, and M. Alsolami, "A Dual-Input Full-Bridge Current-Source Isolated DC / DC Converter Based on Quasi-SwitchedCapacitor Circuit for Photovoltaic Systems with Energy Storage," in Applied Power Electronics Conference and Exposition (APEC), 2015.

[8] O. Bomboir, P. Bleus, F. Milstein, T. Joannès, P. Stassain, and C. Geuzaine, "Red Electrical Devils by CE+T Technical Approach Document," 2015. [Online]. Available: https://www.littleboxchallenge.com/pdf/finalists/56568-Tech.pdf

[9] D. Bortis, D. Neumayr, and J. W. Kolar, " np-Pareto optimization and comparative evaluation of inverter concepts considered for the GOOGLE Little Box Challenge," in Workshop on Control and Modeling for Power Electronics (COMPEL), 2016.
[10] Y. Wu, "Paralleling high-speed GaN power HEMTs for quadrupled power output," in Applied Power Electronics Conference and Exposition (APEC), 2013.

[11] R. Ramachandran and M. Nymand, "Experimental Demonstration of a 98.8Efficient Isolated DC-DC GaN Converter," IEEE Transactions on Industrial Electronics, vol. 64, no. 11, pp. 9104-9113, Nov 2017.

[12] Z. Wang, Y. Wu, J. Honea, and L. Zhou, "Paralleling GaN HEMTs for Diode-free Bridge Power Converters," in Applied Power Electronics Conference and Exposition (APEC), 2015.

[13] J. S. Lai, C.-y. Lin, Y.-c. Liu, L. Zhang, and X. Zhao, "Design Optimization for Ultrahigh Efficiency Buck Regulator using Wide Bandgap Devices," in Energy Conversion Congress and Exposition (ECCE), 2015.

[14] D. Derix, R. Freiche, C. Schöner, and A. Hensel, "Comparison of continuous and transition mode in a PV-booster with GaN- transistors and switching frequencies up to $250 \mathrm{kHz}$ Keywords," in European Conference on Power Electronics and Applications (EPE), 2015.

[15] A. Avila, A. Garcia-Bediaga, F. Gonzalez, X. Jorda, X. Perpina, and A. Rujas, "Thermal performance analysis of GaN-based high-power converters," in IEEE European Conference on Power Electronics and Applications (EPE'18 ECCE Europe), 2018.

[16] R. Hou, J. Xu, and D. Chen, "A multivariable turn-on/turn-off switching loss scaling approach for high-voltage GaN HEMTs in a hard-switching half-bridge configuration," in IEEE Workshop on Wide Bandgap Power Devices and Applications (WiPDA), Oct 2017, pp. 171-176.

[17] S. Bandyopadhyay and J. Morroni, "Quasi-square wave convertersmodeling and performance benefits of $\mathrm{GaN}$ over Silicon," in IEEE Applied Power Electronics Conference and Exposition (APEC), 2017.

[18] M. Kasper, R. M. Burkart, G. Deboy, and J. W. Kolar, "Zvs of power mosfets revisited," IEEE Transactions on Power Electronics, vol. 31, no. 12, pp. 8063-8067, Dec 2016. 